\title{
BILATERAL RISK SHARING WITH HETEROGENEOUS BELIEFS AND EXPOSURE CONSTRAINTS
}

BY

TiM J. BOONEN(iD) AND MARIO GHOSSOUB (D)

\begin{abstract}
This paper studies bilateral risk sharing under no aggregate uncertainty, where one agent has Expected-Utility preferences and the other agent has Rankdependent utility preferences with a general probability distortion function. We impose exogenous constraints on the risk exposure for both agents, and we allow for any type or level of belief heterogeneity. We show that Pareto-optimal risk-sharing contracts can be obtained via a constrained utility maximization under a participation constraint of the other agent. This allows us to give an explicit characterization of optimal risk-sharing contracts. In particular, we show that an optimal risk-sharing contract contains allocations that are monotone functions of the likelihood ratio, where the latter is obtained from Lebesgue's Decomposition Theorem.
\end{abstract}

\section{KEYWORDS}

Risk sharing, Pareto optimality, heterogeneous beliefs, probability distortion, exposure constraints.

JEL codes: C02, D86, G22.

\section{INTRODUCTION}

Bilateral risk sharing is a risk transfer and reallocation mechanism popularized by the prevalence of over-the-counter trading, that is, direct trading between two parties without the supervision of an exchange. This paper examines a situation in which a decision maker (DM), such as a risk manager, is able to construct a contract to transfer risk to another institution (the counterparty). Since the seminal work of Borch (1962) and Wilson (1968), risk sharing is an important problem in economics, finance, and actuarial science. Our focus here is on bilateral risk sharing initiated by the DM, who seeks to maximize 
his/her expected utility (EU) under subjective probabilities. The counterparty is allowed to have different beliefs regarding the underlying risk's probability distribution and is endowed with a flexible class of rank-dependent utility (RDU) preferences (Quiggin, 1982, 1991, 1993) generated by a probability distortion. We do not impose restrictions on the shape of the probability distortion, and we allow in particular for both strongly risk-averse (in the sense of aversion to mean-preserving spreads) and strongly risk-seeking preferences. ${ }^{1}$ We also allow for preferences that overweight extreme (good and bad) risks via an inverse-Sshaped distortion, as in Cumulative Prospect Theory (Tversky and Kahneman, 1992). Both the DM and the counterparty are subject to constraints on their risk-absorbing capacity. In other words, we impose ex ante liability constraints that state that even when a firm assigns a low or zero probability to certain events, it is not able to pay a nearly infinite amount of capital to the other agent.

While the notion of a competitive equilibrium is a popular tool and solution concept in risk-sharing problems, it requires the assumption that individuals regard the prices of goods or consumption bundles as being independent of their own choices, and is thus suitable for markets with many agents (e.g., Arrow and Debreu, 1954). If there are only two agents, this assumption is very strong. Consequently, we focus instead on the weaker concept of Pareto optimality. We show that Pareto-optimal risk-sharing contracts can be obtained by a maximization of the DM's objective function under a participation constraint of the counterparty.

Risk sharing received considerable attention in markets with aggregate uncertainty, since the seminal work of Borch (1962), Wilson (1968), Gerber (1978), Bühlmann and Jewell (1979), and Kaluszka (2004). In such markets, trading a state-contingent payoff is interpreted as hedging rather than betting. Our focus is on risk sharing without aggregate risk, which is the case when the sum of all risk in the market is risk-free before and after the risksharing contract is determined (as in Billot et al., 2000, 2002 or Chateauneuf et al., 2000, for instance). In such markets, trading a state-contingent payoff is interpreted as betting rather than hedging. Our key contribution in this paper is to examine the effect of belief heterogeneity on risk sharing. The two agents may have different beliefs due to asymmetric information, or difficulty in estimating the distribution with limited data. Heterogeneity of beliefs gained considerable interest in economics and finance. For a nonexhaustive list of references that study heterogeneous beliefs in financial markets, we refer to Gollier (2007), David (2008), Chen et al. (2012), and Simsek (2013a,b). Previous approaches to risk sharing with heterogeneous beliefs include Wilson (1968) and Boonen et al. (2017), but these approaches rely on EU preferences and agreements about zero-probability events. Moreover, if all agents are endowed with cash-invariant utilities with different reference probabilities, existence of Pareto-optimal risk-sharing contracts is studied by Acciaio and Svindland (2009). Our approach is more general, as it allows for a very general form of disagreement about probabilities. We allow in particular for singularity 
between the beliefs, that is, disagreement about zero-probability events. In optimal (re)insurance contract design, optimal insurance with divergent beliefs is studied by Boonen (2016), Ghossoub (2016, 2017, 2019a), and Chi (2019), for instance. These approaches impose particular conditions on the type or level of disagreement about probabilities, unlike this paper.

The literature on risk sharing has hitherto examined situations in which the preferences of all agents belong to the same class. For instance, Borch (1962), Wilson (1968), Gerber (1978), Bühlmann and Jewell (1979), Kaluszka (2004), and Aase (1993, 2010) study the EU case, while Jouini et al. (2008) and Ludkovski and Young (2009) study dual utilities (as in Yaari, 1987), or more generally, law-invariant monetary utility functions. Moreover, Tsanakas and Christofides (2006), Xia and Zhou (2016), Jin et al. (2019), and Boonen et al. (2018) study the case of RDU. As an exception, Boonen (2017) studies Pareto-optimal risk sharing with both expected and dual utilities. All of these approaches impose assumptions that ensure that the optimal contracts are comonotonic. ${ }^{2}$ For instance, some authors assume that the beliefs regarding the underlying distribution are homogeneous and the distortions are convex. Moreover, all these optimal solutions are implicit and aim either at characterizing existence of a solution or at providing a solution algorithm. In this paper, we provide an explicit description of an optimal bilateral risk-sharing contract. Interestingly, this contract is not necessarily comonotonic, but it is a monotone function of the likelihood ratio. Existence of this likelihood ratio follows from Lebesgue's Decomposition Theorem.

If agents have homogeneous beliefs and are both averse to mean-preserving spreads, then we show that it is always optimal for both agents not to hold a risky position after risk is transferred from the DM to the counterparty. Billot et al. $(2000,2002)$ and Chateauneuf et al. (2000) show that this also holds in risk sharing with multiple priors, when the agents' sets of priors have a nonempty intersection. We show that optimality of a deterministic position after risk sharing needs no longer to be true when either (i) beliefs are heterogeneous, or (ii) the counterparty is endowed with a general (nonconvex) probability distortion. We characterize optimal risk-sharing contracts for any type or level of belief heterogeneity and any probability distortion function, and we provide an explicit description of the optimal risk-sharing contract for the DM subject to a participation constraint of the counterparty. It has a simple two-part structure: the DM receives a maximal wealth transfer on an event to which the counterparty assigns zero probability, and an explicit solution on the complement of this event. Therefore, the risk-sharing contract can depend on a "sunspot" if the agents disagree on the likelihood of a sunspot occurrence. This concept of a sunspot as an extrinsic random variable is introduced by Cass and Shell (1983), and the existence of sunspot equilibria with Choquet-Expected Utility (CEU) is first shown by Tallon (1998).

The rest of this paper is organized as follows. Section 2 discusses the agents' preferences and introduces the constrained risk-sharing problem. Section 3 examines the relationship between the constrained risk-sharing problem and 
Pareto optimality. Section 4 provides a solution, as well as a description of optimal risk-sharing contracts. Section 5 examines the special cases of dual-utility preferences and expected-utility preferences for the counterparty, respectively. Section 6 concludes. Related analysis and the proofs are presented in the Appendices.

\section{Model Setup}

\subsection{Preferences of the agents}

Let $(S, \Sigma)$ be a measurable space, and let $B(\Sigma)$ be the vector space of all bounded, $\mathbb{R}$-valued, and $\Sigma$-measurable functions on $(S, \Sigma)$.

We assume that there are two agents who seek a risk-sharing arrangement. We propose a maximization of the utility of one agent under a generic participation constraint of the other agent. As we will show in Section 3, this formulation is consistent with the criterion of Pareto optimality in our setting.

The DM is subject to an original risk $X_{1} \in B(\Sigma)$ and the counterparty is subject to a risk $X_{2} \in B(\Sigma)$, where the realizations are interpreted as losses. A key assumption in this paper is that there is no aggregate risk (as in Billot et al., 2000, 2002; Chateauneuf et al., 2000, or Ghirardato and Siniscalchi, 2018), which implies that $X_{1}+X_{2}=c \in \mathbb{R}$. Trading is therefore seen as betting rather than as hedging. For instance, consider a situation where two traders meet and engage in Over-The-Counter trading. The background wealth (initial endowments) of the agents consist of all previous bilateral contracts in their portfolios. Thus, as previous contracts have been bought/sold by the two traders, the initial endowments sum up to zero. Now, suppose that these traders wish to reallocate risk in order to gain jointly from belief heterogeneity. In the literature on risk sharing, it is common to focus on homogeneous beliefs, and to consider an exogenously given aggregate risk. In such situations, Paretooptimal risk allocations are typically comonotonic with this aggregate risk (e.g., Boonen et al., 2018). This does not allow for betting (e.g., put options) against the aggregate risk. In order to isolate the effect of heterogeneous beliefs on Pareto-optimal risk allocations, we keep the aggregate risk deterministic.

A risk-sharing contract is a pair $\left(\hat{X}_{1}, \hat{X}_{2}\right) \in B(\Sigma) \times B(\Sigma)$ such that $\hat{X}_{1}+$ $\hat{X}_{2}=X_{1}+X_{2}=c$. The risk $\hat{X}_{1}$ is subtracted from the initial wealth of the DM, and the risk $\hat{X}_{2}$ is subtracted from the initial wealth of the counterparty, both without financial frictions. The DM has initial wealth $W_{0}^{1} \in \mathbb{R}$, and his/her total state-contingent wealth after risk sharing is the random variable $W \in B(\Sigma)$ defined by

$$
W(s):=W_{0}^{1}-\hat{X}_{1}(s), \forall s \in S .
$$

As in Ghossoub (2017, 2019a), we assume that the DM has preferences over future random wealth admitting a subjective EU representation. The DM's 
preferences induce a utility function $\hat{u}_{1}: \mathbb{R} \rightarrow \mathbb{R}$ and a subjective probability measure $P$ on $(S, \Sigma)$. The DM is risk averse, such that his/her utility function $\hat{u}_{1}$ satisfies the following commonly used assumption.

Assumption 2.1. The DM's utility function $\hat{u}_{1}$ is increasing, ${ }^{3}$ strictly concave, continuously differentiable, and satisfies the Inada conditions $\lim _{x \rightarrow-\infty} \hat{u}_{1}^{\prime}(x)=+\infty$ and $\lim _{x \rightarrow+\infty} \hat{u}_{1}^{\prime}(x)=0$.

We assume that the DM maximizes

$$
\int \hat{u}_{1}(W) d P=\int \hat{u}_{1}\left(W_{0}^{1}-\hat{X}_{1}\right) d P .
$$

The DM will seek to maximize this objective function under a participation constraint of the counterparty. The counterparty's preferences induce a probability measure $Q$ on $(S, \Sigma)$ and a utility function $\hat{u}_{2}: \mathbb{R} \rightarrow \mathbb{R}$. We assume that the counterparty distorts this probability measure $Q$ using a distortion function $T$. Thus, the counterparty is endowed with RDU preferences (Quiggin, 1982, 1991, 1993), which admit a representation in terms of a Choquet integral. ${ }^{4} \mathrm{EU}$ and dual utility are special cases of RDU preferences, and these two special cases will be discussed in more detail in Section 5. We assume that the participation constraint of the counterparty is given by

$$
\int \hat{u}_{2}\left(W_{0}^{2}-\hat{X}_{2}\right) d T \circ Q \geq V_{0},
$$

where $W_{0}^{2} \in \mathbb{R}$ is the counterparty's initial nonrandom wealth, and $V_{0} \in \mathbb{R}$ is the counterparty's reservation utility. As in the $\varepsilon$-constraint method for multiobjective optimization (e.g., Cohon, 1978 and Miettinen, 1999), our aim is to span the set of Pareto-optimal contracts by allowing $V_{0}$ to be flexible. ${ }^{5}$ The probability distortion function $T$ and utility function $\hat{u}_{2}$ satisfy the following assumption.

Assumption 2.2. The probability distortion function $T:[0,1] \rightarrow[0,1]$ is such that $T(0)=0, T(1)=1$, and $T$ is absolutely continuous and increasing. Moreover, the utility function $\hat{u}_{2}$ is increasing, continuously differentiable, and (weakly) concave.

If the distortion function $T$ is convex and $\hat{u}_{2}$ is concave, Chew et al. (1987) show that the counterparty is averse to mean-preserving spreads. In parts of the literature (e.g., Amarante et al., 2015), an RDU preference representation is sometimes seen as a special case of CEU, in which the agent's nonadditive measure (sometimes called a capacity) $v$ is a distortion of a probability measure $(v=T \circ \mu$, for some probability measure $\mu$ ). In this case, convexity (resp. concavity) of the distortion function $T$ yields convexity (resp. concavity) of 
the capacity $v$. In CEU, a convex capacity reflects ambiguity-aversion, while a concave capacity reflects ambiguity-seeking behavior.

\subsection{The constrained demand problem}

To ensure existence of optimal solutions, we impose a lower limit on the expost wealth for both the DM and the counterparty. Specifically, we assume that

$$
W_{0}^{1}-\hat{X}_{1}(s) \geq a, \quad W_{0}^{2}-\hat{X}_{2}(s) \geq b, \quad \forall s \in S,
$$

where $a, b \in \mathbb{R}$ are exogenously given. Note that this is equivalent to assuming exogenous upper bounds on risk exposure $\hat{X}_{i}$, for both the DM and the counterparty. Infinite losses are not feasible in reality due to limited liability, even when an agent assigns zero-probability to these events. Agents are not able to pay out more than a given amount. These bounds are not necessarily the same for the two agents. The wealth of the DM cannot be smaller than $a$, so that we impose $W_{0}^{1}-\hat{X}_{1}(s) \geq a$ for all $s \in S$. Likewise, the wealth of the counterparty cannot fall below $b$, so that $W_{0}^{2}-\hat{X}_{2}(s) \geq b$ for all $s \in S$.

The DM's problem is that of finding a risk-sharing contract that maximizes his/her subjective EU of terminal wealth, subject to the participation constraint of the counterparty, and to the constraints that the ex post wealth satisfies the given lower bounds in Equation (2.3). This is formalized in the following problem.

\section{Problem 2.3.}

$\sup _{\hat{X}_{1}, \hat{X}_{2} \in B(\Sigma)}\left\{\int \hat{u}_{1}\left(W_{0}^{1}-\hat{X}_{1}\right) d P: \begin{array}{c}\hat{X}_{1}+\hat{X}_{2}=c, \quad W_{0}^{1}-\hat{X}_{1} \geq a, W_{0}^{2}-\hat{X}_{2} \geq b, \\ \int \hat{u}_{2}\left(W_{0}^{2}-\hat{X}_{2}\right) d T \circ Q \geq V_{0}\end{array}\right\}$.

Define $Y:=\hat{X}_{1}-c, L:=b-W_{0}^{2}$, and $R:=W_{0}^{1}-a-c$, and define the utility functions $u_{1}$ and $u_{2}$ by $u_{1}(x):=\hat{u}_{1}\left(W_{0}^{1}-c+x\right)$ and $u_{2}(x):=\hat{u}_{2}\left(W_{0}^{2}+x\right)$ for $x \in \mathbb{R}$. We can then rewrite Equation (2.3) as

$$
L \leq Y(s) \leq R, \forall s \in S,
$$

and if $\hat{u}_{1}$ and $\hat{u}_{2}$ satisfy Assumptions 2.1 and 2.2, then so do $u_{1}$ and $u_{2}$, respectively. Substituting this into Problem 2.3 yields the following problem reformulation.

\section{Problem 2.4.}

$$
\sup _{Y \in B(\Sigma)}\left\{\int u_{1}(-Y) d P: L \leq Y \leq R, \int u_{2}(Y) d T \circ Q \geq V_{0}\right\} .
$$


Problem 2.4 is the main problem that we study in this paper. Note that the risk $Y$ may have a negative realization; and a lower realization yields a higher wealth for the DM. We will refer to the risk $Y$ as a risk-sharing contract. To rule out trivial situations, we will make the following assumption.

Assumption 2.5. $u_{2}(L) \leq V_{0} \leq u_{2}(R)$.

Indeed, if $V_{0}>u_{2}(R)$ or $L>R$, then Problem 2.4 has no feasible solution satisfying the participation constraint in Equation (2.2), and thus no risk-sharing contract is optimal. Moreover, if $V_{0}<u_{2}(L)$, then any feasible contract satisfies the participation constraint, and thus the solutions are identical to the solutions when $V_{0}=u_{2}(L)$. Note that $V_{0} \geq \int u_{2}\left(-X_{2}\right) d T \circ Q$ ensures individual rationality for the counterparty, and $\int u_{1}(-Y) d P \geq \int u_{1}\left(c-X_{1}\right) d P$ ensures individual rationality for the DM. We do not impose individual rationality constraints ex ante. This setup also allows us to introduce deterministic financial transaction costs, that thus do not depend on the risk-sharing contract $Y$. These deterministic financial transaction costs need then to be added to $V_{0}$.

\subsection{Singularity and the likelihood ratio}

By Lebesgue's Decomposition Theorem (e.g., Aliprantis and Border, 2006, Theorem 10.61) there exists a unique pair $\left(P_{a c}, P_{s}\right)$ of (nonnegative) finite measures on $(S, \Sigma)$ such that $P=P_{a c}+P_{s}$, where

(i) $P_{a c} \ll Q$, that is, for all $B \in \Sigma, Q(B)=0 \Longrightarrow P_{a c}(B)=0$.

(ii) $P_{s} \perp Q$, that is, there exists some $A \in \Sigma$ such that $Q(S \backslash A)=P_{s}(A)=0$, which then implies that $P_{a c}(S \backslash A)=0$ and $Q(A)=Q(S)=1$.

Therefore, by the Radon-Nikodým Theorem (e.g., Aliprantis and Border, 2006, Theorem 13.20) there exists a $Q$-a.s. unique $h \in L^{1}(S, \Sigma, Q)$ such that $h: S \rightarrow[0,+\infty)$ and $P_{a c}(C)=\int_{C} h d Q$, for all $C \in \Sigma$. Moreover, $h$ can be interpreted as a likelihood ratio: $h=\frac{d P_{a c}}{d Q}$, which is also known as Radon-Nikodým derivative. In the rest of this paper, we fix the set $A$ and the random variable $h$.

\section{The Constrained Demand Problem and Pareto Optimality}

In this section, we examine the relationship between the constrained demand problem in Problem 2.4 and Pareto optimality. We first define the concept of Pareto optimality for our constrained risk-sharing problem. 
Definition 3.1. The risk-sharing contract $Y^{*} \in B(\Sigma)$ satisfying Equation (2.4) is Pareto optimal if there does not exist any other risk-sharing contract $Y \in B(\Sigma)$ satisfying Equation (2.4) such that

$$
\int u_{1}(-Y) d P \geq \int u_{1}\left(-Y^{*}\right) d P \text { and } \int u_{2}(Y) d T \circ Q \geq \int u_{2}\left(Y^{*}\right) d T \circ Q,
$$

with at least one strict inequality.

The next result shows that for every Pareto-optimal risk-sharing contract $Y^{*}$, there exists some $V_{0} \in \mathbb{R}$ such that $Y^{*}$ maximizes the objective function given in Equation (2.1) under the participation constraint given in Equation (2.2).

Theorem 3.2. Suppose that Assumptions 2.1 and 2.2 hold. Then the following hold.

(i) If the risk sharing contract $Y^{*} \in B(\Sigma)$ is Pareto optimal, then it solves Problem 2.4 with $V_{0}:=\int u_{2}\left(Y^{*}\right) d T \circ Q$;

(ii) If $Q \ll P_{a c}$, then for a given $V_{0} \in\left[u_{2}(L), u_{2}(R)\right]$ any solution to Problem 2.4 is Pareto optimal;

(iii) If $Q \ll P_{a c}$ and $Y^{*} \in B(\Sigma)$ solves Problem 2.4 for a given $V_{0} \in$ $\left[u_{2}(L), u_{2}(R)\right]$, then $\int u_{2}\left(Y^{*}\right) d T \circ Q=V_{0}$.

While the first result is well known in operations research and optimization (e.g., Miettinen, 1999, Theorem 3.2.2), the two other results necessitate the handling of the intrinsic singularity between the two probability measures. Note that the condition $Q \ll P_{a c}$, which is equivalent to the mutual absolute continuity of $Q$ and $P_{a c}$ (denoted by $Q \sim P_{a c}$ ), is weaker than the condition $Q \ll P$. If $P \perp Q$ (and so $Q \ll P_{a c}$ does not hold), we will show in Section 4 that there exist solutions to Problem 2.4 that are not Pareto optimal (see Propositions 4.2 and 4.3).

Remark 3.3. As a consequence of Theorem 3.2, we obtain that if $Q \ll P_{a c}$, then finding solutions to Problem 2.4 for all $V_{0} \in\left[u_{2}(L), u_{2}(R)\right]$ is equivalent to finding all Pareto-optimal risk-sharing contracts. Note that $Q \ll P_{a c}$ holds when $P=Q, Q \ll P$, or $Q \sim P$ (i.e., $Q$ and $P$ are equivalent ${ }^{6}$ ). Since Pareto optimality does not distinguish the DM and counterparty other than via their preferences, it is also a solution of the dual approach of maximizing the counterparty's utility under a participation constraint of the DM. Thus, if $Q \sim P$, all solutions of Problem 2.4 are also solutions obtained under the dual approach. 


\section{OPTIMAL RISK-SHARING CONTRACTS}

Our main result, Theorem 4.5, provides an explicit description of an optimal risk-sharing contract, for any level of belief divergence. It is precisely the belief divergence and the probability distortion that create room for risk sharing. We show this in the following proposition.

Proposition 4.1. If Assumptions 2.1, 2.2, and 2.5 hold, if $T$ is convex, and if $P=Q$, then the risk-sharing contract $Y^{*} \equiv u_{2}^{-1}\left(V_{0}\right)$ is optimal in Problem 2.4.

Proposition 4.1 implies that if agents share beliefs, and if the counterparty distorts this common belief via a convex distortion function, then the optimal allocation is the full insurance allocation given by $\hat{X}_{1} \equiv u_{2}^{-1}\left(V_{0}\right)+c$ and $\hat{X}_{2} \equiv-u_{2}^{-1}\left(V_{0}\right)$.

Next, we examine another extreme case where $P$ and $Q$ are mutually singular (i.e., $h \equiv 0$ ).

Proposition 4.2. If Assumptions 2.1, 2.2, and 2.5 hold, and if $P \perp Q$, then for any $L \leq \hat{Y} \leq R$ such that $\int_{A} u_{2}(\hat{Y}) d T \circ Q \geq V_{0}$, the risk-sharing contract

$$
Y^{*}:=L \mathbf{1}_{S \backslash A}+\hat{Y} \mathbf{1}_{A}
$$

is optimal in Problem 2.4. Hence, in particular, $P\left(Y^{*}=L\right)=1$.

When the beliefs are mutually singular, it is optimal for the DM to receive the maximum share from the counterparty (that is to receive $-L$ ) for events to which the DM assigns full probability. Proposition 4.2 characterizes a collection of solutions to Problem 2.4. This collection of solutions is larger than the collection of Pareto-optimal risk-sharing contracts, as shown in the following proposition.

Proposition 4.3. If Assumptions 2.1 and 2.2 hold, and if $P \perp Q$, then any Paretooptimal risk-sharing contract is given by

$$
Y^{*}:=Y_{1} \mathbf{1}_{S \backslash A}+Y_{2} \mathbf{1}_{A},
$$

where $Y_{1}=L, P$-a.s., and $Y_{2}=R, Q$-a.s. In other words, $Y^{*}=L, P$-a.s., and $Y^{*}=R, Q-a$.s.

Proposition 4.3 states that if $P \perp Q$, solutions to Problem 2.4 are not necessarily Pareto optimal. In particular, Proposition 4.2 selects a collection of solutions to Problem 2.4, and when $\hat{Y} \mathbf{1}_{A}=R \mathbf{1}_{A}, Q$-a.s., these solutions are Pareto optimal. Note that Theorem 3.2(ii) states that every solution to Problem 
2.4 is Pareto optimal when $Q \ll P_{a c}$, for example, when $P$ and $Q$ are equivalent probability measures.

Next, we state the main result of this paper. To do so, we need some definitions. For the likelihood ratio $h$, we denote by $F_{h, Q}$ the cumulative distribution function with respect to the probability measure $Q$, defined by

$$
F_{h, Q}(t):=Q(\{s \in S: h(s) \leq t\}), \forall t \in \mathbb{R},
$$

and we denote by $F_{h, Q}^{-1}(t)$ the left-continuous inverse of the distribution function $F_{h, Q}$ (i.e., the quantile function of $h$ w.r.t. $Q$ ), defined by

$$
F_{h, Q}^{-1}(t)=\inf \left\{z \in \mathbb{R}: F_{h, Q}(z) \geq t\right\}, \forall t \in[0,1] .
$$

For a real-valued function $f$ on a convex subset of $\mathbb{R}$ containing the interval $[0,1]$, the convex envelope of $f$ on the interval $[0,1]$ is defined as the greatest convex function $g$ on $[0,1]$ such that $g(x) \leq f(x)$, for each $x \in[0,1]$. The construction of the convex envelope with an inverse-S shaped distortion is studied by Ghossoub (2019b) and Wang et al. (2019). We make the following assumption.

Assumption 4.4. The likelihood ratio $h=\frac{d P_{a c}}{d Q}$ is a continuous random variable on the probability space $(S, \Sigma, Q)$ (i.e., $Q \circ h^{-1}$ is nonatomic) such that $F_{h, Q}^{-1}$ is increasing and positive on $[0,1]$.

This assumption states that the likelihood ratio must, in particular, be a continuous random variable with respect to probability measure $Q$, but not necessarily with respect to probability measure $P$.

Theorem 4.5. If Assumptions 2.1, 2.2, 2.5, and 4.4 hold, then the risk-sharing contract

$$
Y^{*}:=\min \left\{\max \left\{L, g^{*}(h)\right\}, R\right\} \mathbf{1}_{A}+L \mathbf{1}_{S \backslash A}
$$

is optimal for Problem 2.4, where:

- $g^{*}(h):=m^{-1}\left(\lambda^{*} \delta^{\prime}\left(\phi\left(1-F_{h, Q}(h)\right)\right)\right)$, which depends on the state of the world only through the likelihood ratio $h=\frac{d P_{a c}}{d Q}$;

- the function $m$ is defined by $m(x):=\frac{u_{1}^{\prime}(-x)}{u_{2}^{\prime}(x)}$;

- $\delta$ is the convex envelope on $[0,1]$ of the function $\Psi$ defined by $\Psi(t):=1$ $T\left(1-\phi^{-1}(t)\right)$, for all $t \in[0,1]$, where $\phi(t):=\int_{0}^{t} F_{h, Q}^{-1}(1-x) d x$, for all $t \in$ $[0,1]$; and, 
- $\lambda^{*}$ is chosen such that $\int u_{2}\left(Y^{*}\right) d T \circ Q=V_{0}$.

Moreover, the function $g^{*}$ is nonincreasing.

Theorem 4.5 is our main result, and it provides an explicit characterization of a solution $Y^{*}$ to Problem 2.4. The DM retains the random wealth $-Y^{*}$ that is comonotonic with $h$ on the event $A$, which means that $-Y^{*}$ is a nondecreasing function of $h$ on $A$; and the random wealth $Y^{*}$ that the counterparty receives is a nonincreasing function of $h$ on $A$. The solutions of Problem 2.4 in Theorem 4.5 lead to solutions of Problem 2.3. Moreover, note that by Theorem 3.2 solutions to Problem 2.4 are Pareto optimal when $Q \ll P_{a c}$.

Example 4.6. Suppose that the modified utility functions of the two agents are of the exponential type:

$$
u_{i}(x)=-\frac{1}{\alpha_{i}} e^{-\alpha_{i} x}, \text { for } x \in \mathbb{R}
$$

for some $\alpha_{i}>0$, where $i=1,2$ and $\alpha_{1} \neq \alpha_{2}$. Then, for $i=1,2$, both $\hat{u}_{i}$ and $u_{i}$ display constant absolute risk aversion, and the absolute risk aversion is the constant function given by

$$
A R A_{i}(x)=-\frac{\hat{u}_{i}^{\prime \prime}(x)}{\hat{u}_{i}^{\prime}(x)}=-\frac{u_{i}^{\prime \prime}(x)}{u_{i}^{\prime}(x)}=\alpha_{i}, \text { for } x \in \mathbb{R} .
$$

We define the aggregate risk aversion in the market as $\alpha_{1}+\alpha_{2}$. Moreover, in this case,

$$
m(x)=e^{\left(\alpha_{1}+\alpha_{2}\right) x}, \text { for } x \in \mathbb{R}
$$

and thus

$$
m^{-1}(y)=\frac{\ln (y)}{\alpha_{1}+\alpha_{2}}, \text { for } y>0 .
$$

Consequently, Theorem 4.5 implies that the risk-sharing contract

$$
Y^{*}:=\min \left\{\max \left\{L, d+\frac{\ln \left(\delta^{\prime}\left(\phi\left(1-F_{h, Q}(h)\right)\right)\right)}{\alpha_{1}+\alpha_{2}}\right\}, R\right\} \mathbf{1}_{A}+L \mathbf{1}_{S \backslash A}
$$

is optimal for Problem 2.4, where $d \in \mathbb{R}$ is chosen such that $\int u_{2}\left(Y^{*}\right) d T$ 。 $Q=V_{0}$.

Now, for each $y>0$,

$$
\lim _{\alpha_{1} \rightarrow+\infty} m^{-1}(y)=\lim _{\alpha_{2} \rightarrow+\infty} m^{-1}(y)=\lim _{\alpha_{1}+\alpha_{2} \rightarrow+\infty} m^{-1}(y)=0 .
$$


Thus, if $\alpha_{1}+\alpha_{2} \rightarrow+\infty$, it follows that $Y^{*}:=u_{2}^{-1}\left(V_{0}\right) \mathbf{1}_{A}+L \mathbf{1}_{S \backslash A}$ is optimal for Problem 2.4. Hence, if the two agents are very risk-averse, an optimal risksharing contract $Y^{*}$ is deterministic on the event $A$. So, on the set $A$, there is no randomness as a result of heterogeneous beliefs. Moreover, for each $y \in(0,1)$,

$$
\lim _{\alpha_{1}+\alpha_{2} \rightarrow 0} m^{-1}(y)=-\infty
$$

and for each $y>1$,

$$
\lim _{\alpha_{1}+\alpha_{2} \rightarrow 0} m^{-1}(y)=+\infty .
$$

Therefore, when $\alpha_{1}+\alpha_{2} \rightarrow 0$, it follows that the optimal risk-sharing solution $Y^{*}$ is very dispersed on the event $A$, based on the sign of $\delta^{\prime}\left(\phi\left(1-F_{h, Q}(h)\right)\right)$. Hence, if the aggregate risk aversion in the market is small, then there will be more betting based on the heterogeneity of beliefs via the likelihood ratio $h$, and vice versa.

\section{Two Special Cases of the Preferences of the Counterparty}

In this section, we discuss two special cases. First, we consider the case where the counterparty is endowed with EU preferences (no distortion), and second we consider the case where the counterparty is endowed with dual utility preferences (linear utility).

\subsection{EU preferences}

The first special case is the case in the absence of a probability distortion. In this case, $T(t)=t$, for all $t \in[0,1]$. Then, $\Psi(t)=1-T\left(1-\phi^{-1}(t)\right)=\phi^{-1}(t)$ for all $t \in[0,1]$. Moreover, $\phi^{\prime}(t)=F_{h, Q}^{-1}(1-t)>0$, and so $\phi$ is increasing. By Assumption $4.4, F_{h, Q}^{-1}$ is increasing and thus $\phi$ is concave. The Inverse Function Theorem implies that $\phi^{-1}$ is convex and increasing. Consequently, $\Psi$ is convex and increasing on $[0,1]$. Hence, $\delta=\Psi$ on $[0,1]$, yielding

$$
\begin{aligned}
\delta^{\prime}\left(\phi\left(1-F_{h, Q}(h)\right)\right) & =\left(\phi^{-1}\right)^{\prime}\left(\phi\left(1-F_{h, Q}(h)\right)\right)=\frac{1}{\phi^{\prime}\left(1-F_{h, Q}(h)\right)} \\
& =\frac{1}{F_{h, Q}^{-1}\left(F_{h, Q}(h)\right)}=\frac{1}{h}, Q \text {-a.s. }
\end{aligned}
$$

As a direct consequence of the above argument, we obtain the following description of optimal risk-sharing contracts by Theorem 4.5. 
Corollary 5.1. If Assumptions 2.1, 2.5, and 4.4 hold, and if $T(t)=t$ for each $t \in[0,1]$, then the risk-sharing contract

$$
Y^{*}:=\min \left\{\max \left\{L, g^{*}(h)\right\}, R\right\} \mathbf{1}_{A}+L \mathbf{1}_{S \backslash A}
$$

is optimal for Problem 2.4, where:

- $g^{*}(h):=m^{-1}\left(\frac{\lambda^{*}}{h}\right)$, which depends on the state of the world only through $h=$ $\frac{d P_{a c}}{d Q}$;

- the function $m$ is defined by $m(x):=\frac{u_{1}^{\prime}(-x)}{u_{2}^{\prime}(x)}$; and,

- $\lambda^{*}$ is chosen such that $\int u_{2}\left(Y^{*}\right) d Q=V_{0}$.

For instance, if $u_{1}$ and $u_{2}$ are both of the exponential type, then Corollary 5.1 implies that the risk-sharing contract

$$
Y^{*}=\min \left\{\max \left\{L, d-\frac{\ln (h)}{\alpha_{1}+\alpha_{2}}\right\}, R\right\} \mathbf{1}_{A}+L \mathbf{1}_{S \backslash A}
$$

is optimal in Problem 2.4, where $d \in \mathbb{R}$ is chosen such that $\int u_{2}\left(Y^{*}\right) d Q=V_{0}$. Note that by Assumptions 2.1 and 2.2, it follows that $\lim _{y \rightarrow \infty} m^{-1}(y)=\infty$. So, for all $s \in S$ such that $h(s)=0$, it follows that $Y^{*}(s)=R$. So, an optimal risksharing contract is such that maximal risk is shifted to the DM. However, the DM assigns zero probability to these events. The counterparty, on the other hand, may assign a nonzero probability to these events.

\subsection{Dual utility preferences}

Our second special case is the case where the counterparty is endowed with dual utility preferences. Then, it holds that $u_{2}^{\prime}(x)=1$ for all $x \in \mathbb{R}$, and thus

$$
m(x)=\frac{u_{1}^{\prime}(-x)}{u_{2}^{\prime}(x)}=u_{1}^{\prime}(-x) .
$$

So, it holds that $m^{-1}(y)=-\left(u_{1}^{\prime}\right)^{-1}(y)$, and hence we readily obtain the following result from Theorem 4.5.

Corollary 5.2. If Assumptions 2.1, 2.2, 2.5, and 4.4 hold, and if $u_{2}^{\prime}(y)=1$ for all $y \in \mathbb{R}$, then the risk-sharing contract

$$
Y^{*}:=\min \left\{\max \left\{L, g^{*}(h)\right\}, R\right\} \mathbf{1}_{A}+L \mathbf{1}_{S \backslash A}
$$


is optimal for Problem 2.4, where:

- $g^{*}(h):=-\left(u_{1}^{\prime}\right)^{-1}\left(\lambda^{*} \delta^{\prime}\left(\phi\left(1-F_{h, Q}(h)\right)\right)\right)$, which depends on the state of the world only through the likelihood ratio $h=\frac{d P_{a c}}{d Q}$;

- $\delta$ is the convex envelope on $[0,1]$ of the function $\Psi$ defined by $\Psi(t):=1$ $T\left(1-\phi^{-1}(t)\right)$, for all $t \in[0,1]$, where $\phi(t):=\int_{0}^{t} F_{h, Q}^{-1}(1-x) d x$, for all $t \in$ $[0,1]$; and,

- $\lambda^{*}$ is chosen such that $\int u_{2}\left(Y^{*}\right) d T \circ Q=V_{0}$.

\section{CONCLUSION}

This paper examined the problem of bilateral risk sharing with no aggregate uncertainty and when there is a fixed lower and upper bounds on the shifted loss. We assumed that the counterparty and the DM may disagree about the likelihoods associated with the state space. The DM maximizes a subjective expected-utility functional, and the counterparty is endowed with RDU preferences, with a general probability distortion function and a possibly different probability measure from that of the DM. We allowed for any type or level of belief heterogeneity.

We showed that a constrained maximization under a participation constraint yields all Pareto-optimal risk-sharing contracts. We provided a full description of an optimal risk-sharing contract. It has a simple two-part structure: maximal risk is shifted on an event to which the counterparty assigns zero probability, and an explicit formulation on the complement of this event. If the counterparty is endowed with expected or dual utility, simpler solutions are obtained.

\section{ACKNOWLEDGMENTS}

Mario Ghossoub acknowledges financial support from the Natural Sciences and Engineering Research Council of Canada (NSERC Grant No. 201803961).

\section{NOTES}

1. In the language of the Choquet-Expected Utility model of Schmeidler (1989), of which RDU is a special case, this would be referred to as ambiguity-aversion or ambiguity-seeking, respectively.

2. Comonotonicity of contracts implies that all contracts are perfect hedges of each other.

3. Throughout this paper, we mean "increasing" in the strictly increasing sense. We use the terminology "nondecreasing" to mean weakly increasing. We use the same convention for decreasing functions. 
4. For any $Z \in B(\Sigma)$, the Choquet integral of $\hat{u}_{2}(Z)$ with respect to $T \circ Q$ is defined as

$$
\begin{aligned}
\int \hat{u}_{2}(Z) d T \circ Q:= & \int_{0}^{+\infty} T\left(Q\left(\left\{s \in S: \hat{u}_{2}(Z(s))>t\right\}\right)\right) d t \\
& +\int_{-\infty}^{0}\left[T\left(Q\left(\left\{s \in S: \hat{u}_{2}(Z(s))>t\right\}\right)\right)-1\right] d t .
\end{aligned}
$$

Moreover, for any $Z \in B(\Sigma)$ and $C \in \Sigma$, we define $\int_{C} Z d T \circ Q$ as $\int_{C} Z d T \circ Q:=\int Z \mathbf{1}_{C} d T \circ Q$.

5. Note that if we choose $V_{0} \geq \int \hat{u}_{2}\left(W_{0}^{2}-X_{2}\right) d T \circ Q$, risk-sharing contracts satisfying Equation (2.2) are individually rational for the counterparty.

6. An important example of this is when $Q$ is obtained from $P$ by an Esscher exponential tilting, as in Bühlmann (1980), for instance.

7. It is immediate to verify that $\{s \in S: Y(s)=L\} \subseteq\{s \in S: \bar{Y}(s)=Y(s)\}$. Now, if $s \in S$ is such that $\bar{Y}(s)=Y(s)$ but $Y(s)>L$, then $L<\bar{Y}(s)=\max \{Y(s)-\varepsilon, L\}$, and hence $\bar{Y}(s)=Y(s)-$ $\varepsilon<Y(s)$, a contradiction. Consequently, $\{s \in S: \bar{Y}(s)=Y(s)\} \subseteq\{s \in S: Y(s)=L\}$, and hence $\{s \in S: \bar{Y}(s)=Y(s)\}=\{s \in S: Y(s)=L\}$. Therefore, $\{s \in S: \bar{Y}(s)<Y(s)\}=\{s \in S: Y(s)>L\}$. Since $P(Y>L)>0$, it follows that $P(\bar{Y}<Y)>0$.

\section{REFERENCES}

AASE, K.K. (1993) Equilibrium in a reinsurance syndicate; existence, uniqueness and characterization. ASTIN Bulletin, 23(2), 185-211.

AASE, K.K. (2010) Existence and uniqueness of equilibrium in a reinsurance syndicate. ASTIN Bulletin, 40(2), 491-517.

ACCIAIO, B. and Svindland, G. (2009) Optimal risk sharing with different reference probabilities. Insurance: Mathematics and Economics, 44(3), 426-433.

Aliprantis, C.D. and Border, K.C. (2006) Infinite Dimensional Analysis, Third Edition. New York: Springer-Verlag.

Amarante, M., Ghossoub, M. and Phelps, E.S. (2015) Ambiguity on the insurer's side: The demand for insurance. Journal of Mathematical Economics, 58, 61-78.

ArRow, K.J. and DeBreU, G. (1954) Existence of an equilibrium for a competitive economy. Econometrica, 22(3), 265-290.

Billot, A., Chateauneuf, A., Gilboa, I. and Tallon, J.M. (2000) Sharing beliefs: Between agreeing and disagreeing. Econometrica, 68(3), 685-694.

Billot, A., Chateauneuf, A., Gilboa, I. and Tallon, J.M. (2002) Sharing beliefs and the absence of betting in the Choquet expected utility model. Statistical Papers, 43(1), 127-136.

BoONEN, T.J. (2016) Optimal reinsurance with heterogeneous reference probabilities. Risks, 4(3), 26.

BOONEN, T.J. (2017) Risk sharing with expected and dual utilities. ASTIN Bulletin, 47(2), 391415.

BoOnen, T.J., De WAEgenaere, A. and Norde, H. (2017) Redistribution of longevity risk: The effect of heterogeneous mortality beliefs. Insurance Mathematics and Economics, 72, 175-188.

Boonen, T.J., LIU, F. and WANG, R. (2018) Competitive Equilibria in a Comonotone Market. Available at SSRN: https://ssrn.com/abstract $=3091424$.

BORCH, K. (1962) Equilibrium in a reinsurance market. Econometrica, 30(3), 424- 44.

BÜHLMANN, H. (1980) An economic premium principle. ASTIN Bulletin, 11(1), 52-60.

BühlmanN, H. and Jewell, W.S. (1979) Optimal risk exchanges. ASTIN Bulletin, 10(3), $243-262$.

CAmbanis, S., Simons, G. and Stout, W. (1976) Inequalities for Ek(X,Y) when the marginals are fixed. Zeitschrift fur Wahrscheinlichkeitstheorie und verwandte Gebiete, 36(4), 285-294.

CAss, D. and Shell, K. (1983) Do sunspots matter? Journal of Political Economy, 91(2), 193-227.

Chateauneuf, A., Dana, R.A. and Tallon, J.M. (2000) Optimal risk-sharing rules and equilibria with Choquet-expected-utility. Journal of Mathematical Economics, 34(2), 191-214. 
Chen, H., Joslin, S. and Tran, N. (2012) Rare disasters and risk sharing with heterogeneous beliefs. Review of Financial Studies, 25(7), 2189-2224.

CHEw, S.H., KARNI, E. and SAFRA, Z. (1987) Risk aversion in the theory of expected utility with rank dependent probabilities. Journal of Economic Theory, 42(2), 370-381.

CHI, Y. (2019) On the optimality of a straight deductible under belief heterogeneity. ASTIN Bulletin, 42(1), 243-262.

CoHON, J.L. (1978) Multiobjective Programming and Planning, Vol. 140. Mathematics in Science and Engineering. New York: Academic Press.

DAVID, A. (2008) Heterogeneous beliefs, speculation, and the equity premium. Journal of Finance, 63(1), 41-83.

Föllmer, H. and Schied, A. (2016) Stochastic Finance: An Introduction in Discrete Time, Fourth Edition. Berlin: Walter de Gruyter.

Gerber, H.U. (1978) Pareto-optimal risk exchanges and related decision problems. ASTIN Bulletin, 10(1), 25-33.

GHIRARDATO, P. and SinisCALCHI, M. (2018) Risk sharing in the small and in the large. Journal of Economic Theory, 175, 730-765.

Ghossoub, M. (2015) Equimeasurable rearrangements with capacities. Mathematics of Operations Research, 40(2), 429-445.

Ghossoub, M. (2016) Optimal insurance with heterogeneous beliefs and disagreement about zero-probability events. Risks, 4(3), 29.

Ghossoub, M. (2017) Arrow's theorem of the deducible with heterogeneous beliefs. North American Actuarial Journal, 21(1), 15-35.

GHossoub, M. (2019a) Budget-constrained optimal insurance with belief heterogeneity. Insurance Mathematics and Economics, 89, 79-91.

GHossoub, M. (2019b) Optimal insurance under rank-dependent expected utility. Insurance: Mathematics and Economics, 87, 51-66.

GOLLIER, C. (2007) Whom should we believe? Aggregation of heterogeneous beliefs. Journal of Risk and Uncertainty, 35(2), 107-127.

He, X., KouwenberG, R. and Zhou, X.Y. (2017) Rank-dependent utility and risk taking in complete markets. SIAM Journal on Financial Mathematics, 8(1), 214-239.

JIN, H., XIA, J. and ZHOU, X.Y. (2019) Arrow-Debreu equilibria for rank-dependent utilities with heterogeneous probability weighting. Mathematical Finance, 29(3), 898-927.

Jouini, E., SCHACHERMAYER, W. and TouZI, N. (2008) Optimal risk sharing for law invariant monetary utility functions. Mathematical Finance, 18(2), 269-292.

KALUSZKA, M. (2004) An extension of the Gerber-Bühlmann-Jewell conditions for optimal risk sharing. ASTIN Bulletin, 34(1), 27-48.

LUDKOVSKI, M. and YounG, V.R. (2009) Optimal risk sharing under distorted probabilities. Mathematics and Financial Economics, 2(2), 87-105.

MiETtinen, K. (1998) Nonlinear Multiobjective Optimization, Vol. 12. International Series in Operations Research and Management Science. New York: Springer Science+Business Media.

MoriguTI, S. (1953) A modification of Schwarz's inequality with applications to distributions. Annals of Mathematical Statistics, 24, 107-113.

QuigGin, J. (1982) A theory of anticipated utility. Journal of Economic Behavior and Organisation, 3(4), 323-343.

QUigGin, J. (1991) Comparative statics for rank-dependent expected utility theory. Journal of Risk and Uncertainty, 4(4), 339-350.

QuigGin, J. (1993) Generalized Expected Utility Theory - The Rank-Dependent Model. Dordrecht: Springer Science+Business Media.

SCHMEIDLER, D. (1989) Subjective probability and expected utility without additivity, Econometrica, 57(3), 571-587.

SimseK, A. (2013a) Belief disagreements and collateral constraints. Econometrica, 81(1), 1-53.

SIMSEK, A. (2013b) Speculation and risk sharing with new financial assets. Quarterly Journal of Economics, 128(3), 1365-1396.

TALLON, J.M. (1998) Do sunspots matter when agents are Choquet-expected-utility maximizers? Journal of Economic Dynamics and Control, 22(3), 357-368.

TSANAKAS, A. and CHRISTOFIDES, N. (2006) Risk exchange with distorted probabilities. ASTIN Bulletin, 36(1), 219. 
TVERsky, A. and KAhneman, D. (1992) Advances in prospect theory: Cumulative representation of uncertainty. Journal of Risk and Uncertainty, 5(4), 297-323.

WANG, R., XU, Z.Q. and ZHOU, X.Y. (2019) Dual utilities on risk aggregation under dependence uncertainty. Finance and Stochastics, 23(4), 1025-1048.

WILSON, R. (1968) The theory of syndicates. Econometrica, 36(1), 119-132.

XIA, J. and ZHOU, X.Y. (2016) Arrow-Debreu equilibria for rank-dependent utilities. Mathematical Finance, 26(3), 558-588.

XU, Z.Q. (2016) A note on the quantile formulation. Mathematical Finance, 26(3), 558-588.

YAARI, M. (1987) The dual theory of choice under risk. Econometrica, 55(1), 95-115.

TIM J. BOONEN (Corresponding author)

Amsterdam School of Economics

University of Amsterdam

Roetersstraat 11, 1018 WB, Amsterdam

The Netherlands

E-Mail:t.j.boonen@uva.nl

MARIO GHOSSOUB

Department of Statistics and Actuarial Science

University of Waterloo

200 University Ave. W., Waterloo

N2L 3G1, Canada

E-Mail:mario.ghossoub@uwaterloo.ca

\section{APPENDIX A. EQUIMEASURABLE REARRANGEMENTS}

Let $(S, \mathcal{G}, \mu)$ be a probability space and let $V \in L^{\infty}(S, \mathcal{G}, \mu)$ be a continuous random variable (i.e., $\mu \circ V^{-1}$ is nonatomic). For each $Z \in L^{\infty}(S, \mathcal{G}, \mu)$, let

$$
F_{Z, \mu}(t)=\mu(\{s \in S: Z(s) \leq t\})
$$

denote the cumulative distribution function of $Z$ with respect to the probability measure $\mu$, and let $F_{Z, \mu}^{-1}(t)$ be the left-continuous inverse of the distribution function $F_{Z, \mu}$ (i.e., the quantile function of $Z$ w.r.t. $\mu$ ), defined by

$$
F_{Z, \mu}^{-1}(t)=\inf \left\{z \in \mathbb{R}: F_{Z, \mu}(z) \geq t\right\}, \forall t \in[0,1] .
$$

Definition A.1. Two functions $Y_{1}, Y_{2} \in B(\Sigma)$ are said to be comonotonic (resp., anticomonotonic) if

$$
\left[Y_{1}(s)-Y_{1}\left(s^{\prime}\right)\right]\left[Y_{2}(s)-Y_{2}\left(s^{\prime}\right)\right] \geq 0(\text { resp. }, \leq 0), \text { for all } s, s^{\prime} \in S .
$$

For instance, if $Y_{1}, Y_{2} \in B(\Sigma)$, and if $Y_{2}$ is of the form $Y_{2}=I \circ Y_{1}$, for some Borelmeasurable function $I$, then $Y_{2}$ is comonotonic with $Y_{1}$ if and only if the function $I$ is nondecreasing. 
Proposition A.2 (Ghossoub, 2015). For any $Y \in L^{\infty}(S, \mathcal{G}, \mu)$, define $\tilde{Y}_{\mu}=F_{Y, \mu}^{-1}$ $\left(1-F_{V, \mu}(V)\right)$. Then,

(i) $Y$ and $\widetilde{Y}_{\mu}$ have the same distribution under $\mu$.

(ii) $\widetilde{Y}_{\mu}$ is anti-comonotonic with $V$.

(iii) For each $L \leq R$, if $L \leq Y \leq R$, then $L \leq \widetilde{Y}_{\mu} \leq R$.

(iv) If $Z^{*}$ is any other element of $L^{\infty}(S, \mathcal{G}, \mu)$ that has the same distribution as $Y$ under $\mu$ and that is anti-comonotonic with $V$, then $Z^{*}=\widetilde{Y}_{\mu}, \mu$-a.s.

$\tilde{Y}_{\mu}$ is called the nonincreasing $\mu$-rearrangement of $Y$ with respect to $V$. Since $\mu \circ V^{-1}$ is nonatomic, it follows that $F_{V, \mu}(V)$ has a uniform distribution over $(0,1)$ (Föllmer and Schied, 2016, Lemma A.25). Letting $U:=F_{V, \mu}(V)$, it follows that $U$ is a random variable on the probability space $(S, \Sigma, \mu)$ with a uniform distribution on $(0,1)$ and that $V=F_{V, \mu}^{-1}(U), \mu$-a.s..

A function $L: \mathbb{R}^{2} \rightarrow \mathbb{R}$ is said to be supermodular if for any $x_{1}, x_{2}, y_{1}, y_{2} \in \mathbb{R}$ with $x_{1} \leq x_{2}$ and $y_{1} \leq y_{2}$, one has

$$
L\left(x_{2}, y_{2}\right)+L\left(x_{1}, y_{1}\right) \geq L\left(x_{1}, y_{2}\right)+L\left(x_{2}, y_{1}\right) .
$$

Equation (A2) then implies that a function $L: \mathbb{R}^{2} \rightarrow \mathbb{R}$ is supermodular if and only if the function $\eta(y):=L(x+z, y)-L(x, y)$ is nondecreasing on $\mathbb{R}$, for any $x \in \mathbb{R}$ and $z \geq 0$.

\section{Example A.3.}

(1) If $g: \mathbb{R} \rightarrow \mathbb{R}$ is concave and $a \in \mathbb{R}$, then the function $L_{1}: \mathbb{R}^{2} \rightarrow \mathbb{R}$ defined by $L_{1}(x, y)=$ $g(a-x+y)$ is supermodular. Moreover, if $g$ is strictly concave, then $L_{1}$ is strictly supermodular.

(2) If $\psi, \phi: \mathbb{R} \rightarrow \mathbb{R}$ are both nonincreasing or both nondecreasing functions, then the function $L_{2}: \mathbb{R}^{2} \rightarrow \mathbb{R}$ defined by $L_{2}(x, y)=\phi(x) \psi(y)$ is supermodular.

Proposition A.4 (Hardy-Littlewood-Pólya Inequality, see Cambanis et al., 1976). Let $Y \in$ $L^{\infty}(S, \mathcal{G}, \mu)$, and let $\widetilde{Y}_{\mu}$ be the nonincreasing $\mu$-rearrangement of $Y$ with respect to $V$. If the function $L$ is supermodular, then

$$
\int L\left(V, \widetilde{Y}_{\mu}\right) d \mu \leq \int L(V, Y) d \mu,
$$

provided the integrals exist.

\section{APPENDIX B. PRoOF OF THEOREM 3.2}

The first statement follows almost directly from Theorem 3.2.2 in Miettinen (1999), where the state space is finite. We provide a short self-contained proof for general probability spaces. Suppose that $Y^{*}$ is Pareto optimal. Then, $L \leq Y^{*} \leq R$, and so $\int u_{2}\left(Y^{*}\right) d T \circ Q \in$ $\left[u_{2}(L), u_{2}(R)\right]$. Suppose that $Y^{*}$ does not solve Problem 2.4 with $V_{0}=\int u_{2}\left(Y^{*}\right) d T$ 。 $Q$. Then, there exist $Y \in B(\Sigma)$ satisfying Equation (2.4) that solves Problem 2.4 with $V_{0}$. 
Therefore,

$$
\int u_{1}(-Y) d P>\int u_{1}\left(-Y^{*}\right) d P \text { and } \int u_{2}(Y) d T \circ Q \geq V_{0}=\int u_{2}\left(Y^{*}\right) d T \circ Q .
$$

Thus, $Y$ is a Pareto improvement over $Y^{*}$, contradicting the Pareto optimality of $Y^{*}$.

We continue with the second statement. Suppose that $Q \ll P_{a c}$, fix $V_{0} \in\left[u_{2}(L), u_{2}(R)\right]$, and let $Y^{*}$ be a solution to Problem 2.4. Suppose that $Y^{*}$ is not Pareto optimal, so that there exist $Y \in B(\Sigma)$ satisfying Equation (2.4) with

$$
\int u_{1}(-Y) d P \geq \int u_{1}\left(-Y^{*}\right) d P, \text { and } \int u_{2}(Y) d T \circ Q \geq \int u_{2}\left(Y^{*}\right) d T \circ Q,
$$

with at least one strict inequality. Then, in particular, $Y$ is feasible for Problem 2.4. If $\int u_{1}(-Y) d P>\int u_{1}\left(-Y^{*}\right) d P$, this contradicts the optimality of $Y^{*}$ for Problem 2.4. Assume then that $\int u_{2}(Y) d T \circ Q>\int u_{2}\left(Y^{*}\right) d T \circ Q$, and let $\varepsilon$ be such that $\int u_{2}(Y-\varepsilon) d T \circ Q=\int u_{2}\left(Y^{*}\right) d T \circ Q$. Then $\varepsilon>0$, by strict monotonicity of $u_{2}$. If $Y=L, Q$-a.s., then $u_{2}(L)=\int u_{2}(Y) d T \circ Q>\int u_{2}\left(Y^{*}\right) d T \circ Q \geq V_{0}$, a contradiction. Hence, $Q(Y>L)>0$ and so $P(Y>L) \geq P_{a c}(Y>L)>0$, since $Q \ll P_{a c}$. Let $\bar{Y}:=$ $\max (L, Y-\varepsilon) \in B(\Sigma)$. Then $L \leq \bar{Y} \leq R, Y-\varepsilon \leq \bar{Y} \leq Y$, and $P(\bar{Y}<Y)>0$. Therefore, since $u_{1}$ is increasing, it follows that

$$
\begin{aligned}
\int u_{1}(-\bar{Y}) d P & =\int_{[\bar{Y}=Y]} u_{1}(-Y) d P+\int_{[\bar{Y}<Y]} u_{1}(-\bar{Y}) d P \\
& >\int_{[\bar{Y}=Y]} u_{1}(-Y) d P+\int_{[\bar{Y}<Y]} u_{1}(-Y) d P \\
& =\int u_{1}(-Y) d P \geq \int u_{1}\left(-Y^{*}\right) d P .
\end{aligned}
$$

Moreover, since $\bar{Y} \geq Y-\varepsilon$ it follows that

$$
\int u_{2}(\bar{Y}) d T \circ Q \geq \int u_{2}(Y-\varepsilon) d T \circ Q=\int u_{2}\left(Y^{*}\right) d T \circ Q \geq V_{0},
$$

where the second inequality follows from the feasibility of $Y^{*}$ for Problem 2.4. Consequently, $\bar{Y}$ is feasible for Problem 2.4, which contradicts the optimality of $Y^{*}$ for Problem 2.4.

We conclude with the proof of the third statement. Suppose that $Q \ll P_{a c}$, fix $V_{0} \in$ $\left[u_{2}(L), u_{2}(R)\right]$, and let $Y^{*}$ be a solution to Problem 2.4. Suppose that $\int u_{2}\left(Y^{*}\right) d T \circ Q>$ $V_{0}$. If $Y=L, Q$-a.s., then $u_{2}(L)=\int u_{2}(Y) d T \circ Q>\int u_{2}\left(Y^{*}\right) d T \circ Q>V_{0}$, a contradiction. Hence, $Q(Y>L)>0$ and so $P(Y>L) \geq P_{a c}(Y>L)>0$, since $Q \ll P_{a c}$. Let $\varepsilon$ be such that $\int u_{2}\left(Y^{*}-\varepsilon\right) d T \circ Q=V_{0}$. Then $\varepsilon>0$. Let $\bar{Y}:=\max \left(L, Y^{*}-\varepsilon\right) \in B(\Sigma)$. Then 
$L \leq \bar{Y} \leq R, Y^{*}-\varepsilon \leq \bar{Y} \leq Y^{*}$, and $P\left(\bar{Y}<Y^{*}\right)>0$. Consequently,

$$
V_{0}=\int u_{2}\left(Y^{*}-\varepsilon\right) d T \circ Q \leq \int u_{2}(\bar{Y}) d T \circ Q \leq \int u_{2}\left(Y^{*}\right) d T \circ Q,
$$

and so $\bar{Y}$ is feasible for Problem 2.4. Moreover, since $u_{1}$ is increasing, it follows that

$$
\begin{aligned}
\int u_{1}(-\bar{Y}) d P & =\int_{\left[\bar{Y}=Y^{*}\right]} u_{1}\left(-Y^{*}\right) d P+\int_{\left[\bar{Y}<Y^{*}\right]} u_{1}(-\bar{Y}) d P \\
& >\int_{\left[\bar{Y}=Y^{*}\right]} u_{1}\left(-Y^{*}\right) d P+\int_{\left[\bar{Y}<Y^{*}\right]} u_{1}\left(-Y^{*}\right) d P \\
& =\int u_{1}\left(-Y^{*}\right) d P,
\end{aligned}
$$

contradicting the optimality of $Y^{*}$ for Problem 2.4. Therefore, $\int u_{2}\left(Y^{*}\right) d T \circ Q=V_{0}$.

\section{APPENDIX C. PRoOf of Proposition 4.1}

It is well known that a concave utility function yields risk-aversion in EU, so that due to the Jensen's inequality we have

$$
\int u_{1}\left(-\int Y d P\right) d P=u_{1}\left(-\int Y d P\right) \geq \int u_{1}(-Y) d P
$$

for all $Y \in B(\Sigma)$. Chew et al. (1987) show that in RDU, a concave utility function with a convex probability distortion function yields aversion to mean-preserving spreads. Hence, in particular, concavity of $u_{2}$ and convexity of $T$ imply that for all $Y \in B(\Sigma)$

$$
\int u_{2}(Y) d P=\int\left(\int u_{2}(Y) d P\right) d T \circ P \geq \int u_{2}(Y) d T \circ P .
$$

Thus, if $Y$ solves Problem 2.4, then so does $Y^{*}:=\int u_{2}(Y) d P$. The rest follows from Theorem 3.2(iii).

\section{APPENDIX D. PROOF OF PROPOSITION 4.2}

First, for any $A \in \Sigma$ and $Y \in B(\Sigma)$, let $\int_{A} Y d T \circ Q:=\int Y 1_{A} d T \circ Q$, where $1_{A}$ is the indicator function of $A$.

Proposition D.1. $\int Y d T \circ Q=\int_{A} Y d T \circ Q$, for each $Y \in B(\Sigma)$ such that the integrals are defined. 
Proof. Since, $Q(A)=1$ and since $Q$ is monotone, nonnegative, and additive, we have for each $B \in \Sigma$,

$$
\begin{aligned}
T \circ Q(B) & =T(Q(B))=T(Q((B \cap A) \cup(B \cap(S \backslash A)))) \\
& =T(Q(B \cap A)+Q(B \cap(S \backslash A)))=T(Q(B \cap A))=T \circ Q(B \cap A) .
\end{aligned}
$$

Therefore, it follows from Equation (D1) that

$$
T \circ Q(B \cup(S \backslash A))=T \circ Q((B \cup(S \backslash A)) \cap A)=T \circ Q(B \cap A)=T \circ Q(B) .
$$

Consequently, for any $Y \in B(\Sigma)$, setting $B_{t}:=\{s \in S: Y(s)>t\}$ for all $t \in \mathbb{R}$, it follows that

$$
\begin{aligned}
& \int_{A} Y d T \circ Q=\int_{0}^{+\infty} T \circ Q\left(\left\{s \in S: Y(s) 1_{A}(s)>t\right\}\right) d t \\
& \quad+\int_{-\infty}^{0}\left[T \circ Q\left(\left\{s \in S: Y(s) 1_{A}(s)>t\right\}\right)-1\right] d t \\
& =\int_{0}^{+\infty} T \circ Q(\{s \in A: Y(s)>t\}) d t+\int_{-\infty}^{0}[T \circ Q(\{s \in A: Y(s)>t\} \cup(S \backslash A))-1] d t \\
& =\int_{0}^{+\infty} T \circ Q(\{s \in A: Y(s)>t\}) d t+\int_{-\infty}^{0}[T \circ Q(\{s \in A: Y(s)>t\})-1] d t \\
& =\int_{0}^{+\infty} T \circ Q\left(A \cap B_{t}\right) d t+\int_{-\infty}^{0}\left[T \circ Q\left(A \cap B_{t}\right)-1\right] d t \\
& =\int_{0}^{+\infty} T \circ Q\left(B_{t}\right) d t+\int_{-\infty}^{0}\left[T \circ Q\left(B_{t}\right)-1\right] d t=\int Y d T \circ Q .
\end{aligned}
$$

If $P \perp Q$, then $P=P_{S}, P_{a c} \equiv 0$, and $h \equiv 0$. In this case, $P(A)=0$ and $Q(A)=1$. Choose any $\hat{Y} \in B(\Sigma)$ such that $L \leq \hat{Y} \leq R$ and $\int_{A} u_{2}(\hat{Y}) d T \circ Q \geq V_{0}$, and define $Y^{*} \in B(\Sigma)$ by

$$
Y^{*}:=L \mathbf{1}_{S \backslash A}+\hat{Y} \mathbf{1}_{A} .
$$

Then $L \leq Y^{*} \leq R$ by construction, and Proposition D.1 implies that

$$
\int u_{2}\left(Y^{*}\right) d T \circ Q=\int_{A} u_{2}\left(Y^{*}\right) d T \circ Q=\int_{A} u_{2}(\hat{Y}) d T \circ Q \geq V_{0} .
$$

Hence, $Y^{*}$ is feasible for Problem 2.4. Suppose, by way of contradiction, that $Y^{*}$ is not optimal for Problem 2.4. Then, there exist $Z \in B(\Sigma)$ such that $L \leq Z \leq R, \int u_{2}(Z) d T \circ Q \geq$ $V_{0}$, and $\int u_{1}(-Z) d P>\int u_{1}\left(-Y^{*}\right) d P$. Since $L \leq Z$ and $P(S \backslash A)=1$, it follows that $\int u_{1}(-Z) d P \leq \int u_{1}(-L) d P=\int_{S \backslash A} u_{1}(-L) d P=\int_{S \backslash A} u_{1}\left(-Y^{*}\right) d P=\int u_{1}\left(-Y^{*}\right) d P$, which is a contradiction. Hence, $Y^{*}$ is optimal for Problem 2.4. 


\section{APPENDIX E. Proof of Proposition 4.3}

Let $Z \in B(\Sigma)$ be such that $L \leq Z \leq R$. Then $Y^{*}(s)=R \geq Z(s)$, for $Q$-a.e. $s \in A$, and $u_{1}\left(-Y^{*}(s)\right)=u_{1}(-L) \geq u_{1}(-Z(s))$, for $P$-a.e. $s \in S \backslash A$. Since $P(S \backslash A)=1$, it follows that

$$
\int u_{1}\left(-Y^{*}\right) d P=\int_{S \backslash A} u_{1}\left(-Y^{*}\right) d P=u_{1}(-L) \geq \int_{S \backslash A} u_{1}(-Z) d P=\int u_{1}(-Z) d P .
$$

Moreover, it follows from Proposition D. 1 and $Q(A)=1$ that

$$
\begin{aligned}
\int u_{2}\left(Y^{*}\right) d T \circ Q & =\int_{A} u_{2}\left(Y^{*}\right) d T \circ Q=u_{2}(R) \\
& =\int_{A} u_{2}(R) d T \circ Q \geq \int_{A} u_{2}(Z) d T \circ Q=\int u_{2}(Z) d T \circ Q .
\end{aligned}
$$

Thus, there cannot exist a $Z \in B(\Sigma)$ such that $L \leq Z \leq R$ with

$$
\int u_{1}(-Z) d P \geq \int u_{1}\left(-Y^{*}\right) d P \text { and } \int u_{2}(Z) d T \circ Q \geq \int u_{2}\left(Y^{*}\right) d T \circ Q,
$$

with at least one strict inequality. Consequently, $Y^{*}$ is Pareto optimal.

Furthermore, suppose that $\hat{Y} \in B(\Sigma)$ is another Pareto-optimal risk-sharing contract. Then $L \leq \hat{Y} \leq R$. Hence, in particular, $\int u_{1}(-\hat{Y}) d P \leq u_{1}(-L)$. Moreover,

$$
u_{2}(R) \geq \int u_{2}(\hat{Y}) d T \circ Q=\int_{A} u_{2}(\hat{Y}) d T \circ Q \geq u_{2}(L) .
$$

Suppose, by way of contradiction, that $P(N)>0$, where $N:=\{s \in S: L<\hat{Y}(s)\}$. Then $\int_{N} u_{1}(-\hat{Y}) d P<\int_{N} u_{1}(-L) d P$, by definition of $N$ and strict monotonicity of $u_{1}$. Thus,

$$
\begin{aligned}
\int u_{1}(-L) d P & >\int_{N} u_{1}(-\hat{Y}) d P+\int_{S \backslash N} u_{1}(-L) d P \\
& =\int_{N} u_{1}(-\hat{Y}) d P+\int_{S \backslash N} u_{1}(-\hat{Y}) d P=\int u_{1}(-\hat{Y}) d P .
\end{aligned}
$$

Since the deterministic contract $L$ is feasible, this contradicts the Pareto optimality of $\hat{Y}$. Hence, $\hat{Y}=L, P$-a.s. Now, suppose, by way of contradiction, that $Q(\{s \in A: R>\hat{Y}(s)\})>0$. Then, there exists a $t_{0}<u_{2}(R)$ such that

$$
Q\left(\left\{s \in A: u_{2}(R)>t\right\}\right)=1>Q\left(\left\{s \in A: u_{2}(\hat{Y}(s))>t\right\}\right), \forall t \in\left[t_{0}, u_{2}(R)\right) .
$$

Then by strict monotonicity of $T$ and $u_{2}$, we have

$$
T\left(Q\left(\left\{s \in A: u_{2}(\hat{Y}(s))>t\right\}\right)\right) \leq T\left(Q\left(\left\{s \in A: u_{2}(R)>t\right\}\right)\right), \forall t \in \mathbb{R},
$$


and

$$
T\left(Q\left(\left\{s \in A: u_{2}(\hat{Y}(s))>t\right\}\right)\right)<T\left(Q\left(\left\{s \in A: u_{2}(R)>t\right\}\right)\right), \forall t \in\left[t_{0}, u_{2}(R)\right) .
$$

Thus,

$$
\begin{aligned}
& \int u_{2}(\hat{Y}) d T \circ Q=\int_{A} u_{2}(\hat{Y}) d T \circ Q \\
& =\int_{[0,+\infty) \cap\left[t_{0}, u_{2}(R)\right)} T\left(Q\left(\left\{s \in A: u_{2}(\hat{Y}(s))>t\right\}\right)\right) d t \\
& \quad+\int_{(-\infty, 0) \cap\left[t_{0}, u_{2}(R)\right)}\left[T\left(Q\left(\left\{s \in A: u_{2}(\hat{Y}(s))>t\right\}\right)\right)-1\right] d t \\
& \quad+\int_{\left.[0,+\infty) \backslash t_{0}, u_{2}(R)\right)} T\left(Q\left(\left\{s \in A: u_{2}(\hat{Y}(s))>t\right\}\right)\right) d t \\
& \quad+\int_{(-\infty, 0) \backslash\left[t_{0}, u_{2}(R)\right)}\left[T\left(Q\left(\left\{s \in A: u_{2}(\hat{Y}(s))>t\right\}\right)\right)-1\right] d t \\
& <\int_{0}^{+\infty} T\left(Q\left(\left\{s \in A: u_{2}(R)>t\right\}\right)\right) d t+\int_{-\infty}^{0}\left[T\left(Q\left(\left\{s \in A: u_{2}(R)>t\right\}\right)\right)-1\right] d t \\
& =\int_{A} u_{2}(R) d T \circ Q=u_{2}(R) .
\end{aligned}
$$

Since $Y=R$ is feasible, this contradicts the Pareto optimality of $\hat{Y}$. Hence, $\hat{Y}=R, Q$-a.s.

\section{APPENDIX F. PROOF OF THEOREM 4.5}

Consider the following two problems:

Problem F.1.

$$
\sup _{Y \in B(\Sigma)}\left\{\int_{A} u_{1}(-Y) d P: L \leq Y \leq R, \int u_{2}(Y) d T \circ Q \geq V_{0}\right\} .
$$

\section{Problem F.2.}

$$
\sup _{Y \in B(\Sigma)}\left\{\int_{S \backslash A} u_{1}(-Y) d P: L \mathbf{1}_{S \backslash A} \leq Y \mathbf{1}_{S \backslash A} \leq R \mathbf{1}_{S \backslash A}, \int_{S \backslash A} u_{2}(Y) d T \circ Q=0\right\} .
$$

Since the function $u_{1}$ is continuous by Assumption 2.1, it is bounded on any closed and bounded subset of $\mathbb{R}$. Therefore, since the range of $Y$ is bounded, the supremum of each of the above two problems is finite when their feasibility sets are nonempty. Moreover, the constant function $Y \equiv u_{2}^{-1}\left(V_{0}\right)$ is feasible for Problem F.1 by Assumption 2.5, and so Problem F.1 has a nonempty feasibility set. 
Lemma F.3. The random variable $Y^{*}:=L \mathbf{1}_{S \backslash A}$ is optimal for Problem F.2.

Proof. The feasibility of $Y^{*}=L \mathbf{1}_{S \backslash A}$ for Problem F.2 is clear. To show optimality, let $Y$ be any feasible solution for Problem F.2. Then for each $s \in S \backslash A, L \leq Y(s) \leq R$. Therefore, since $u_{1}$ is increasing, we have for each $s \in S \backslash A, u_{1}(-Y(s)) \leq u_{1}(-L)=u_{1}\left(-Y^{*}(s)\right)$. Thus,

$$
\int_{S \backslash A} u_{1}(-Y) d P \leq \int_{S \backslash A} u_{1}\left(-Y^{*}\right) d P=u_{1}(-L) P(S \backslash A) .
$$

Lemma F.4. If $Y_{1}^{*}$ is optimal for Problem F.1, then $Y^{*}:=Y_{1}^{*} \mathbf{1}_{A}+L \mathbf{1}_{S \backslash A}$ is optimal for Problem 2.4.

Proof. By the feasibility of $Y_{1}^{*}$ for Problem F.1, we have $L \leq Y_{1}^{*} \leq R$ and $\int u_{2}\left(Y_{1}^{*}\right)$ $d T \circ Q \geq V_{0}$. Therefore, $L \leq Y^{*} \leq R$, and Proposition D.1 yields

$$
\int u_{2}\left(Y^{*}\right) d T \circ Q=\int_{A} u_{2}\left(Y^{*}\right) d T \circ Q=\int_{A} u_{2}\left(Y_{1}^{*}\right) d T \circ Q=\int u_{2}\left(Y_{1}^{*}\right) d T \circ Q \geq V_{0} .
$$

Hence, $Y^{*}$ is feasible for Problem 2.4. To show optimality of $Y^{*}$ for Problem 2.4, let $Z$ be feasible for Problem 2.4. Then $Z$ is feasible for Problem F.1, and so the optimality of $Y_{1}^{*}$ for Problem F.1 yields $\int_{A} u_{1}\left(-Y^{*}\right) d P=\int_{A} u_{1}\left(-Y_{1}^{*}\right) d P \geq \int_{A} u_{1}(-Z) d P$. Moreover, $L \mathbf{1}_{S \backslash A} \leq Z \mathbf{1}_{S \backslash A} \leq R \mathbf{1}_{S \backslash A}$, since $L \leq Z \leq R$. Additionally, by Proposition D.1, we have

$$
\int_{S \backslash A} u_{2}(Z) d T \circ Q=\int u_{2}(Z) \mathbf{1}_{S \backslash A} d T \circ Q=\int_{A} u_{2}(Z) \mathbf{1}_{S \backslash A} d T \circ Q=0 .
$$

Hence, $Z$ is feasible for Problem F.2. Thus, by Lemma F.3, $u_{1}(-L) P(S \backslash A)=$ $\int_{S \backslash A} u_{1}(-L) d P \geq \int_{S \backslash A} u_{1}(-Z) d P$, and so we obtain

$$
\begin{aligned}
\int u_{1}\left(-Y^{*}\right) d P & =\int_{A} u_{1}\left(-Y^{*}\right) d P+\int_{S \backslash A} u_{1}\left(-Y^{*}\right) d P \\
& =\int_{A} u_{1}\left(-Y_{1}^{*}\right) d P+u_{1}(-L) P(S \backslash A) \\
& \geq \int_{A} u_{1}(-Z) d P+\int_{S \backslash A} u_{1}(-Z) d P=\int u_{1}(-Z) d P .
\end{aligned}
$$

Consequently, $Y^{*}$ is optimal for Problem 2.4.

For all $Z \in B(\Sigma)$, we have

$$
\int Z d P=\int_{A} Z h d Q+\int_{S \backslash A} Z d P_{S}=\int_{A} Z h d Q+\int_{S \backslash A} Z d P=\int Z h d Q+\int_{S \backslash A} Z d P,
$$


where the second-to-last equality follows from the fact that $\int_{S \backslash A} Z d P_{S}=\int_{S \backslash A} Z d P$, since $P_{a c}(S \backslash A)=0$, and the last equality follows from the fact that $Q(S \backslash A)=0$. Consequently, for all $Z \in B(\Sigma), \int_{A} Z d P=\int_{A} Z h d Q=\int Z h d Q$. Hence, we can rewrite Problem F.1 as:

\section{Problem F.5.}

$$
\sup _{Y \in B(\Sigma)}\left\{\int u_{1}(-Y) h d Q: L \leq Y \leq R, \int u_{2}(Y) d T \circ Q \geq V_{0}\right\} .
$$

We will solve Problem F.1 using a quantile reformulation. For that purpose, let $\mathcal{Q}^{*}$ denote the collection of all $Q$-quantile functions $f$ that satisfy $L \leq f(t) \leq R$ for all $t \in(0,1)$, that is,

$$
\mathcal{Q}^{*}=\{f:(0,1) \rightarrow[L, R] \mid f \text { is nondecreasing and left-continuous }\},
$$

and consider the following problem:

Problem F.6.

$$
\sup _{f \in \mathcal{Q}^{*}}\left\{\int_{0}^{1} u_{1}(-f(t)) F_{h, Q}^{-1}(1-t) d t: \int_{0}^{1} T^{\prime}(1-t) u_{2}(f(t)) d t \geq V_{0}\right\} .
$$

Lemma F.7. If $f^{*}$ is optimal for Problem F.6, then $Y^{*}=f^{*}\left(1-F_{h, Q}(h)\right)$ is optimal for Problem F.5 and anti-comonotonic with $h$.

Proof. First, note that since $Q \circ h^{-1}$ is nonatomic by Assumption 4.4, it follows that $F_{h, Q}(h)$ has a uniform distribution over $(0,1)$ (Föllmer and Schied, 2016, Lemma A.25), that is, $Q\left(\left\{s \in S: F_{h, Q}(h)(s) \leq t\right\}\right)=t$ for each $t \in(0,1)$. Letting $\widetilde{U}:=F_{h, Q}(h)$, it follows that $\widetilde{U}$ is a random variable on the probability space $(S, \Sigma, Q)$ with a uniform distribution on $(0,1)$ and that $h=F_{h, Q}^{-1}(\widetilde{U}), Q$-a.s.

Let $f^{*}$ be optimal for Problem F. 6 and $Y^{*}=f^{*}(1-\widetilde{U})$. Then, since $f^{*} \in \mathcal{Q}^{*}$, it follows that $F_{Y^{*}, Q}^{-1}=f^{*}$ and $L \leq Y^{*} \leq R$. Moreover, by monotonicity of $u_{2}$, Fubini's Theorem yields

$$
\int u_{2}\left(Y^{*}\right) d T \circ Q=\int_{0}^{1} T^{\prime}(1-t) u_{2}\left(f^{*}(t)\right) d t \geq V_{0},
$$

where the inequality follows from the feasibility of $f^{*}$ for Problem F.6. Hence, $Y^{*}$ is feasible for Problem F.5.

To show optimality of $Y^{*}$ for Problem F.5, let $Y$ be any other feasible solution for Problem F.5 and $F_{Y, Q}^{-1}$ its quantile function. Then $\widetilde{Y}:=F_{Y, Q}^{-1}(1-\widetilde{U})$ is the nonincreasing rearrangement of $Y$ with respect to $h$, and so $\tilde{Y}$ is feasible solution for Problem F.5, by properties of the rearrangement (Proposition A.2). Moreover, since the function $u_{1}$ is increasing, it follows that the map $L: \mathbb{R}^{2} \rightarrow \mathbb{R}$ defined by $L(x, y):=-u_{1}(-y) x$ is supermodular (see 
Example A.3). Consequently, by Proposition A.4, it follows that

$$
-\int u_{1}(-\tilde{Y}) h d Q=\int L(h, \tilde{Y}) d Q \leq \int L(h, Y) d Q=-\int u_{1}(-Y) h d Q,
$$

that is, $\int u_{1}(-\tilde{Y}) h d Q \geq \int u_{1}(-Y) h d Q$. Since $Y$ is feasible for Problem F.5, it follows that $F_{Y, Q}^{-1}$ is feasible for Problem F.6. Therefore,

$$
\begin{aligned}
& \int u_{1}(-Y) d P=\int u_{1}(-Y) h d Q \\
& \quad \leq \int u_{1}(-\widetilde{Y}) h d Q=\int u_{1}\left(-F_{Y, Q}^{-1}(1-\widetilde{U})\right) h d Q \\
& \quad=\int u_{1}\left(-F_{Y, Q}^{-1}(1-\widetilde{U})\right) F_{h, Q}^{-1}(\widetilde{U}) d Q \\
& \quad=\int_{0}^{1} u_{1}\left(-F_{Y, Q}^{-1}(1-t)\right) F_{h, Q}^{-1}(t) d t \leq \int_{0}^{1} u_{1}\left(-f^{*}(1-t)\right) F_{h, Q}^{-1}(t) d t \\
& =\int u_{1}\left(-f^{*}(1-\widetilde{U})\right) F_{h, Q}^{-1}(\widetilde{U}) d Q=\int u_{1}\left(-Y^{*}\right) h d Q=\int u_{1}\left(-Y^{*}\right) d P,
\end{aligned}
$$

where the second inequality follows from the optimality of $f^{*}$ for Problem F.6. Therefore, $Y^{*}$ is optimal for Problem F.5.

Fix $f \in \mathcal{Q}^{*}$ and define the function $\phi$ by $\phi(t):=\int_{0}^{t} F_{h, Q}^{-1}(1-x) d x$, so that

$$
\int_{0}^{1} u_{1}(-f(t)) F_{h, Q}^{-1}(1-t) d t=\int_{0}^{1} u_{1}(-f(t)) d \phi(t) .
$$

Letting $v(t)=\phi^{-1}(t)$ and $z=v^{-1}(t)$, we obtain

$$
\begin{aligned}
\int_{0}^{1} u_{1}(-f(t)) F_{h, Q}^{-1}(1-t) d t & =\int_{0}^{1} u_{1}(-f(t)) d v^{-1}(t)=\int_{0}^{1} u_{1}(-f(v(z))) d z \\
& =\int_{0}^{1} u_{1}(-q(t)) d t,
\end{aligned}
$$

where $q(t):=f(v(t))$. Define the function $\Psi$ on $[0,1]$ by

$$
\Psi(t):=1-T(1-v(t))=1-T\left(1-\phi^{-1}(t)\right) .
$$

Then, $\Psi^{\prime}(t)=T^{\prime}(1-v(t)) v^{\prime}(t)$, and so

$$
\int_{0}^{1} u_{2}(f(t)) T^{\prime}(1-t) d t=\int_{0}^{1} u_{2}(f(v(z))) T^{\prime}(1-v(z)) d v(z)=\int_{0}^{1} u_{2}(q(t)) \Psi^{\prime}(t) d t .
$$

Consider now the following problem: 


\section{Problem F.8.}

$$
\sup _{q \in \mathcal{Q}^{*}}\left\{\int_{0}^{1} u_{1}(-q(t)) d t: \int_{0}^{1} u_{2}(q(t)) \Psi^{\prime}(t) d t \geq V_{0}\right\} .
$$

Lemma F.9. If $q^{*}$ is a solution of Problem F.8, then $f^{*}:=q^{*} \circ \phi$ is a solution of Problem F.6.

Proof. Suppose $q^{*}$ is optimal for Problem F.8. Since $q^{*} \in \mathcal{Q}^{*}$, it follows that $f^{*} \in \mathcal{Q}^{*}$. Moreover, using the variable $z=v^{-1}(t)$, where $v=\phi^{-1}$ and $\phi$ are as defined above, we have

$$
\begin{gathered}
\int_{0}^{1} T^{\prime}(1-t) u_{2}\left(f^{*}(t)\right) d t=\int_{0}^{1} T^{\prime}(1-v(z)) u_{2}\left(f^{*}(v(z))\right) d v(z)=\int_{0}^{1} u_{2}\left(f^{*}(v(t))\right) \Psi^{\prime}(t) d t \\
=\int_{0}^{1} u_{2}\left(q^{*}(\phi(v(t)))\right) \Psi^{\prime}(t) d t=\int_{0}^{1} u_{2}\left(q^{*}(t)\right) \Psi^{\prime}(t) d t \geq V_{0},
\end{gathered}
$$

where the last inequality follows from the feasibility of $q^{*}$ for Problem F.8. Therefore, $f^{*}$ is feasible for Problem F.6.

To show optimality of $f^{*}$ for Problem F.6, let $f$ be any other feasible solution for Problem F.6, and let $q:=f \circ v$. Since $f$ is feasible for Problem F.6, it is nondecreasing, leftcontinuous, and satisfies, for all $t \in(0,1), L \leq f(t) \leq R$. Therefore, since $v$ is increasing and continuous (by the Inverse Function Theorem), $q$ is nondecreasing, left-continuous, and satisfies, for all $t \in(0,1), L \leq q(t)=f(v(t)) \leq R$. Therefore, $q \in \mathcal{Q}^{*}$. Furthermore,

$\int_{0}^{1} u_{2}(q(t)) \Psi^{\prime}(t) d t=\int_{0}^{1} u_{2}(f(v(z))) T^{\prime}(1-v(z)) d v(z)=\int_{0}^{1} T^{\prime}(1-t) u_{2}(f(t)) d t \geq V_{0}$,

where the last inequality follows from the feasibility of $f$ for Problem F.6. Thus, $q$ is feasible for Problem F.8, and hence $\int_{0}^{1} u_{1}(-q(t)) d t \leq \int_{0}^{1} u_{1}\left(-q^{*}(t)\right) d t$. Thus, using the variable $z=v^{-1}(t)$, we obtain

$$
\begin{aligned}
\int_{0}^{1} & u_{1}(-f(t)) F_{h, Q}^{-1}(1-t) d t=\int_{0}^{1} u_{1}(-f(t)) d v^{-1}(t)=\int_{0}^{1} u_{1}(-f(v(z))) d z \\
& =\int_{0}^{1} u_{1}(-q(t)) d t \\
& \leq \int_{0}^{1} u_{1}\left(-q^{*}(t)\right) d t=\int_{0}^{1} u_{1}\left(-f^{*}(v(z))\right) d z=\int_{0}^{1} u_{1}\left(-f^{*}(t)\right) d v^{-1}(t) \\
& =\int_{0}^{1} u_{1}\left(-f^{*}(t)\right) \phi^{\prime}(t) d t \\
& =\int_{0}^{1} u_{1}\left(-f^{*}(t)\right) F_{h, Q}^{-1}(1-t) d t
\end{aligned}
$$

Therefore, $f^{*}$ is optimal for Problem F.6. 
In light of Lemma F.9, we turn our attention to solving Problem F.8. In order to do that, we will use a similar methodology to the one used by $\mathrm{Xu}$ (2016), but adapted to the present setting. First, we recall the following result, due to He et al. (2017, Appendix A).

Lemma F.10 (He et al., 2017). Let $f$ be a continuous real-valued function on a nonempty convex subset of $\mathbb{R}$ containing the interval $[0,1]$, and let $g$ be its convex envelope on the interval $[0,1]$. Then,

(1) $g$ is continuous and convex on $[0,1]$;

(2) $g(0)=f(0)$ and $g(1)=f(1)$;

(3) for all $x \in[0,1], g(x) \leq f(x)$;

(4) $g$ is affine on $\{x \in[0,1]: g(x)<f(x)\}$.

\section{Moreover,}

(5) Iff is increasing, then so is $g$;

(6) Iff is continuously differentiable on $(0,1)$, then $g$ is continuously differentiable on $(0,1)$.

The following lemma is a direct consequence of Theorem 1 of Moriguti (1953).

Lemma F.11 (Moriguti, 1953). Let $\delta$ be the convex envelope of $\Psi$ on $[0,1]$. Then for any $q \in \mathcal{Q}^{*}$,

$$
\int_{0}^{1} u_{2}(q(t)) \Psi^{\prime}(t) d t \leq \int_{0}^{1} u_{2}(q(t)) \delta^{\prime}(t) d t
$$

Now, consider the following problem.

Problem F.12.

$$
\sup _{q \in \mathcal{Q}^{*}}\left\{\int_{0}^{1} u_{1}(-q(t)) d t: \int_{0}^{1} u_{2}(q(t)) \delta^{\prime}(t) d t \geq V_{0}\right\} .
$$

We first solve Problem F.12 and then show that the solution is also optimal for Problem F.8.

Lemma F.13. If $q^{*} \in \mathcal{Q}^{*}$ satisfies

(1) $\int_{0}^{1} \delta^{\prime}(t) u_{2}\left(q^{*}(t)\right) d t=V_{0}$;

(2) there exists some $\lambda>0$ such that for all $t \in(0,1)$,

$$
q^{*}(t)=\underset{L \leq y \leq R}{\arg \max }\left\{u_{1}(-y)+\lambda u_{2}(y) \delta^{\prime}(t)\right\},
$$

then $q^{*}$ is optimal for Problem F.12.

Proof. Let $q^{*} \in \mathcal{Q}^{*}$ be such that the two conditions above are satisfied. Then $q^{*}$ is feasible for Problem F.12. To show optimality, let $q \in \mathcal{Q}^{*}$ be any feasible solution for Problem F.12. Then, by definition of $q^{*}$, it follows that for each $t$, 


$$
u_{1}\left(-q^{*}(t)\right)-u_{1}(-q(t)) \geq \lambda\left[\delta^{\prime}(t) u_{2}(q(t))-\delta^{\prime}(t) u_{2}\left(q^{*}(t)\right)\right] .
$$

Hence, $\int_{0}^{1} u_{1}\left(-q^{*}(t)\right) d t-\int_{0}^{1} u_{1}(-q(t)) d t \geq \lambda\left[\int_{0}^{1} \delta^{\prime}(t) u_{2}(q(t)) d t-V_{0}\right] \geq 0$. Consequently, it follows that $\int_{0}^{1} u_{1}\left(-q^{*}(t)\right) d t \geq \int_{0}^{1} u_{1}(-q(t)) d t$.

Lemma F.14. For each $\lambda>0$, define the function $q_{\lambda}^{*}$ by

$$
\left.q_{\lambda}^{*}(t):=\min \left\{\max \left\{L, m^{-1}\left(\lambda \delta^{\prime}(t)\right)\right)\right\}, R\right\}, \text { for all } t \in(0,1),
$$

where the function $m$ is defined by

$$
m(x):=\frac{u_{1}^{\prime}(-x)}{u_{2}^{\prime}(x)}
$$

Then,

(1) for each $\lambda>0, q_{\lambda}^{*} \in \mathcal{Q}^{*}$;

(2) there exists $a \lambda^{*}>0$ such that $\int_{0}^{1} \delta^{\prime}(t) u_{2}\left(q_{\lambda^{*}}^{*}(t)\right) d t=V_{0}$;

(3) for all $t \in(0,1)$ and all $\lambda>0, q_{\lambda}^{*}(t)=\underset{L \leq y \leq R}{\arg \max }\left\{u_{1}(-y)+\lambda u_{2}(y) \delta^{\prime}(t)\right\}$.

Proof. Assumptions 2.1 and 2.2 imply that $u_{1}$ and $u_{2}$ are increasing and continuously differentiable. Moreover, $u_{1}^{\prime}$ is decreasing and $u_{2}^{\prime}$ is nonincreasing. Therefore, the function $m$ is continuously differentiable and

$$
m^{\prime}(x)=\frac{-u_{1}^{\prime \prime}(-x) u_{2}^{\prime}(x)-u_{1}^{\prime}(-x) u_{2}^{\prime \prime}(x)}{\left(u_{2}^{\prime}(x)\right)^{2}}>0 .
$$

Hence, the function $m$ is continuous and increasing. Consequently, it is invertible, and its inverse is also increasing, by the Inverse Function Theorem. Thus, for each $\lambda>0$, the convexity and continuity of $\delta$ imply that the function $g_{\lambda}^{*}=m^{-1}\left(\lambda \delta^{\prime}\right)$ is continuous and increasing. Therefore, for each $\lambda>0, q_{\lambda}^{*} \in \mathcal{Q}^{*}$.

Now, for each $t \in(0,1)$ and $\lambda>0$, the concavity of $u_{1}$ and $u_{2}$ yield the concavity of the function $y \mapsto \mathcal{M}_{\lambda, t}(y):=u_{1}(-y)+\lambda u_{2}(y) \delta^{\prime}(t)$, since $\delta^{\prime}(t) \geq 0$. Therefore, first-order conditions yield a global maximum of the function $\mathcal{M}_{\lambda, t}$ at $y^{*}=m^{-1}\left(\lambda \delta^{\prime}(t)\right)$. If $y^{*} \leq L$, then $L=\arg \max \mathcal{M}_{\lambda, t}(y)$. If $y^{*} \geq R$, then $R=\arg \max \mathcal{M}_{\lambda, t}(y)$. Therefore, for all $t \in(0,1)$ $L \leq y \leq R$ $L \leq y \leq R$ and all $\lambda>0, q_{\lambda}^{*}(t)=\underset{L \leq y \leq R}{\arg \max } \mathcal{M}_{\lambda, t}(y)$, where the function $q_{\lambda}^{*}$ is as in Equation (F3).

Finally, the existence of $\lambda^{*}>0$ such that $\int_{0}^{1} \delta^{\prime}(t) u_{2}\left(q_{\lambda^{*}}^{*}(t)\right) d t=V_{0}$ follows from Assumption 2.5, the monotonicity and continuity properties of $\delta^{\prime}$ (see Lemma F.10), and from the Intermediate Value Theorem. 
Therefore, Lemmata F.11, F.13, and F.14 imply that for any $\lambda>0$ and any $q \in \mathcal{Q}^{*}$,

$$
\begin{aligned}
& \int_{0}^{1}\left[u_{1}(-q(t))+\lambda u_{2}(q(t)) \Psi^{\prime}(t)\right] d t \leq \int_{0}^{1} u_{1}(-q(t)) d t+\lambda \int_{0}^{1} u_{2}(q(t)) \delta^{\prime}(t) d t \\
& \quad \leq \int_{0}^{1}\left[u_{1}\left(-q_{\lambda}^{*}(t)\right)+\lambda u_{2}\left(q_{\lambda}^{*}(t)\right) \delta^{\prime}(t)\right] d t,
\end{aligned}
$$

where $q_{\lambda}^{*}$ is as in Equation (F3). Now, for all $\lambda>0$, we have $q_{\lambda}^{*} \in \mathcal{Q}^{*}$ by Lemma F.14, and

$$
q_{\lambda}^{*}(t)= \begin{cases}L & \text { if } m^{-1}\left(\lambda \delta^{\prime}(t)\right) \leq L, \\ m^{-1}\left(\lambda \delta^{\prime}(t)\right) & \text { if } L<m^{-1}\left(\lambda \delta^{\prime}(t)\right)<R, \\ R & \text { if } m^{-1}\left(\lambda \delta^{\prime}(t)\right) \geq R .\end{cases}
$$

Thus,

$$
d q_{\lambda}^{*}(t)= \begin{cases}0 & \text { if } m^{-1}\left(\lambda \delta^{\prime}(t)\right)<L, \\ \lambda\left(m^{-1}\right)^{\prime}\left(\lambda \delta^{\prime}(t)\right) d \delta^{\prime}(t) & \text { if } L<m^{-1}\left(\lambda \delta^{\prime}(t)\right)<R, \\ 0 & \text { if } m^{-1}\left(\lambda \delta^{\prime}(t)\right)>R .\end{cases}
$$

Letting $\mathcal{D}:=\{t \in[0,1]: \delta(t) \neq \Psi(t)\}=\{t \in[0,1]: \delta(t)<\Psi(t)\}$, it follows that for any $\lambda>0$,

$$
\int_{0}^{1}[\Psi(t)-\delta(t)] d u_{2}\left(q_{\lambda}^{*}(t)\right)=\int_{\mathcal{D}}[\Psi(t)-\delta(t)] d u_{2}\left(q_{\lambda}^{*}(t)\right)
$$

But, since $\delta$ is affine on $\mathcal{D}, d \delta^{\prime}=0$ on $\mathcal{D}$, and it follows from Equation (F4) that $d q_{\lambda}^{*}(t)=$ 0 on $\mathcal{D}$, for all $\lambda>0$. Consequently, $\int_{0}^{1}[\Psi(t)-\delta(t)] d u_{2}\left(q_{\lambda}^{*}(t)\right)=0$. Therefore, applying Fubini's Theorem yields $0=\int_{0}^{1}[\Psi(t)-\delta(t)] d u_{2}\left(q_{\lambda}^{*}(t)\right)=\int_{0}^{1} u_{2}\left(q_{\lambda}^{*}(x)\right)\left[\Psi^{\prime}(x)-\right.$ $\left.\delta^{\prime}(x)\right] d x$. Consequently, $\int_{0}^{1} u_{2}\left(q_{\lambda}^{*}(t)\right) \Psi^{\prime}(t) d t=\int_{0}^{1} u_{2}\left(q_{\lambda}^{*}(t)\right) \delta^{\prime}(t) d t$. Therefore, for all $\lambda>0$ and all $q \in \mathcal{Q}^{*}$,

$$
\begin{aligned}
\int_{0}^{1}\left[u_{1}\left(-u_{2}(q(t))\right)+\lambda u_{2}(q(t)) \Psi^{\prime}(t)\right] d t & \leq \int_{0}^{1}\left[u_{1}\left(-u_{2}\left(q_{\lambda}^{*}(t)\right)\right)+\lambda u_{2}\left(q_{\lambda}^{*}(t)\right) \delta^{\prime}(t)\right] d t \\
& =\int_{0}^{1}\left[u_{1}\left(-u_{2}\left(q_{\lambda}^{*}(t)\right)\right)+\lambda u_{2}\left(q_{\lambda}^{*}(t)\right) \Psi^{\prime}(t)\right] d t .
\end{aligned}
$$

Hence, if $\lambda^{*}$ is chosen such that $\int_{0}^{1} u_{2}\left(q_{\lambda^{*}}^{*}(t)\right) \Psi^{\prime}(t) d t=V_{0}$, then the optimal solution to Problem F.8 is given by $q_{\lambda^{*}}^{*}$. Thus, by Lemmata F.7, F.9, F.13, and F.14, the function

$$
Y^{*}=q_{\lambda^{*}}^{*}(\phi(1-\widetilde{U}))
$$

is optimal for Problem F.5 and anti-comonotonic with $h$, where

- $\widetilde{U}:=F_{h, Q}(h)$ is uniformly distributed on $(0,1)$ for the measure $Q$; 
- for all $\left.t \in[0,1], q_{\lambda^{*}}^{*}(t)=\min \left\{\max \left\{L, m^{-1}\left(\lambda^{*} \delta^{\prime}(t)\right)\right)\right\}, R\right\}$;

- the function $m$ is defined by $m(x):=\frac{u_{1}^{\prime}(-x)}{u_{2}^{\prime}(x)}$;

- $\delta$ is the convex envelope on $[0,1]$ of the function $\Psi$ defined by $\Psi(t):=1-T\left(1-\phi^{-1}(t)\right)$, for all $t \in[0,1]$, where $\phi(t):=\int_{0}^{t} F_{h, Q}^{-1}(1-x) d x$, for all $t \in[0,1]$; and,

- $\lambda^{*}$ is chosen such that $\int_{0}^{1} u_{2}\left(q_{\lambda^{*}}^{*}(t)\right) \Psi^{\prime}(t) d t=V_{0}$, and so $\int u_{2}\left(Y^{*}\right) d T \circ Q=V_{0}$.

Consequently, by Lemma F.4, $Y^{*} \mathbf{1}_{A}+L \mathbf{1}_{S \backslash A}$ is optimal for Problem 2.4.

Finally, the fact that $g^{*}$ is nonincreasing is a direct consequence of the convexity of $\delta$, the fact that $\lambda^{*}>0$, and the fact that $m, \phi$, and $F_{h, Q}$ are all increasing (see the proof of Lemma F.14 and Assumption 4.4). This concludes the proof of Theorem 4.5. 